\title{
An Interpretation of Completeness and Basu's Theorem
}

\author{
E. L. LEHMANN*
}

In order to obtain a statistical interpretation of completeness of a sufficient statistic $T$, an attempt is made to characterize this property as equivalent to the condition that all ancillary statistics are independent of $T$. For technical reasons, this characterization is not correct, but two correct versions are obtained in Section 3 by modifying either the definition of ancillarity or of completeness. The light this throws on the meaning of completeness is discussed in Section 4. Finally, Section 5 reviews some of the difficulties encountered in models that lack completeness.

KEY WORDS: Ancillary statistic; Basu's theorem; Completeness; Conditioning; Unbiased estimation.

\section{INTRODUCTION}

Let $X$ be a random observable distributed according to a distribution from the family $\mathscr{P}=\left\{P_{\theta}, \theta \in \Omega\right\}$. Recall the following definitions.

A statistic $V=V(X)$ is ancillary if its distribution does not depend on $\theta$.

A statistic $T=T(X)$ is sufficient if the conditional distribution of $X$ given $T$ does not depend on $\theta$.

These two concepts are complementary. If the rest of the data is discarded, a sufficient statistic by itself retains all the information about $\theta$ contained in the data; an ancillary statistic by itself contains no information about $\boldsymbol{\theta}$.

Since sufficiency reduces the data without loss of information, a sufficient statistic is of greatest interest when it is minimal, that is, when it provides the greatest reduction of the data. Formally, a sufficient statistic is minimal if it is a function of every other sufficient statistic. Although exceptions are possible (Landers and Rogge 1972; Pitcher 1975), minimal sufficient statistics typically exist and are easy to construct (Bahadur 1954; Lehmann and Scheffé 1950).

A sufficient statistic $T$ is complete if

$$
\left.E_{\theta} f(T)=0 \text { for all } \theta \Rightarrow f(t)=0 \text { (a.e. } \mathscr{P}\right),
$$

and is boundedly complete if (1.1) holds for all bounded

* E. L. Lehmann is Professor, Department of Statistics, University of California, Berkeley, CA 94720 . Research was supported in part by National Science Foundation Grant MCS79-03716 and Office of Naval Research Contract N00014-75-C-0444. The author wishes to thank the referee for his very careful reading of two versions of the manuscript, and in particular for his discovery of an error in the original version of Theorem 3. $f$. A necessary condition for $T$ to be complete or boundedly complete is that it is minimal.

The properties of minimality and completeness are of a rather different nature. Under mild assumptions a minimal sufficient statistic exists and whether or not $T$ is minimal then depends on the choice of $T$. On the other hand, existence of a complete sufficient statistic is equivalent to the completeness of the minimal sufficient statistic, and hence is a property of the model $\mathscr{P}$. We shall say that a model is complete if its minimal sufficient statistic is complete.

It has been pointed out in the literature that definition (1.1) is purely technical and does not suggest a direct statistical interpretation. Since various aspects of statistical inference become particularly simple for complete models, one would expect this concept to carry statistical meaning. It is the principal aim of this article to attempt a statistical characterization of models which are complete or boundedly complete. In the last section, we shall briefly survey some of the difficulties presented by incomplete models.

\section{AN EXAMPLE AND BASU'S THEOREM}

In order to get some insight into the problem, consider the following example.

Example 1. Let $X_{1}, \ldots, X_{n}$ be iid with density $f\left(x_{i}\right.$ $-\theta$ ), and contrast what happens when $f$ is normal, exponential, or uniform on the one hand, and in most other cases, for example, when $f$ is logistic, Cauchy, or double exponential on the other. In the fifst type of situation the data can be reduced without loss of information to a small number of sufficient statistics: one for the normal $(\bar{X})$ and exponential $\left(X_{(1)}\right)$, and two for the uniform $\left(X_{(1)}, X_{(n)}\right)$, where $X_{(1)}<\cdots<X_{(n)}$ denote the order statistics. In the second type of situation, the set of $n$ order statistics is minimal sufficient. (Intermediate cases are of course possible, as is seen, for example, by considering $f(x-\theta)$ $=C e^{-(x-\theta)^{2 k}}$.)

As will be seen later, this difference is related to the following property of the ancillary information. In any location problem, the $n-1$ differences $Y_{i}=X_{(n)}-X_{(i)}$ $(i=1, \ldots, n-1)$ constitute an $(n-1)$-dimensional ancillary statistic. In the normal and exponential cases, the $Y$ 's are independent of the minimal sufficient statistic

(c) Journal of the American Statistical Association June 1981, Volume 76, Number 374 Theory and Methods Section 
and hence carry no information about $\theta$ either by themselves or in conjunction with the minimal sufficient statistic. On the other hand, when the $n$ order statistics are minimal, the differences $Y_{i}$-although still ancillary-are functions of the minimal sufficient statistic. Thus, sufficiency has not been successful in squeezing out the ancillary material. By themselves, the $Y^{\prime}$ s carry no information but in conjunction with the rest of the data they do.

The uniform case is intermediate. Here the $Y$ 's are not independent of the minimal sufficient statistic, but the ratios $Z_{i}=Y_{i} / Y_{1}(i=2, \ldots, n-1)$ are. If we represent the ordered sample by

$$
\left(\frac{1}{2}\left[X_{(1)} \times X_{(n)}\right], \quad Y_{1}=X_{(n)}-X_{(1)}, Z_{2}, \ldots, Z_{n-1}\right)
$$

we see that the minimal sufficient statistic $T=\left(X_{(1)}, X_{(n)}\right)$ has pushed out the $n-2 Z$ 's, which carry no information by themselves or in conjunction with the rest of the data, but not $Y_{1}$.

Note that the minimal sufficient statistic is complete in the normal and exponential cases, but not in the other four cases. This suggests that completeness of a minimal sufficient statistic $T$ may be associated with the ability of $T$ completely to rid itself of the ancillary part of the data by making it independent of $T$. As a further illustration of this idea, consider a sample from a uniform distribution on $\left(\theta_{1}, \theta_{2}\right)$ where now both $\theta$ 's are unknown. The minimal sufficient statistic continues to be $T=\left(X_{(1)}\right.$, $\left.X_{(n)}\right)$. However, $Y_{1}$ is no longer ancillary, although the $Z$ 's continue to be so. In the present case, $T$ is more successful in discarding ancillary material than it was in the one-parameter case, and $T$ is now complete.

These comments are closely related to a theorem of Basu $(1955,1958)$, which states that when a sufficient statistic $T$ is boundedly complete, then all ancillary statistics are independent of $T$. This would provide the desired characterization of complete models as those in which the minimal sufficient statistic is completely successful in discarding all ancillary material, if the converse were also true, that is, if the independence of all ancillary statistics from a sufficient statistic implied that $T$ were boundedly complete. Unfortunately this is not correct (see Sec. 3, Ex. 2) but it contains a germ of truth.

\section{SOME ADAPTATIONS OF BASU'S THEOREM}

The converse of Basu's theorem fails because ancillarity is concerned with the whole distribution of a statistic, while completeness is a property dealing only with expectation. We shall now show that correct versions of the converse can be obtained by replacing either ancillarity with the corresponding first-order property or completeness with a condition reflecting the whole distribution.

Let us call a real-valued statistic $V=V(X)$ first-order ancillary if $E_{\theta}(V)$ is independent of $\theta$. (A first-order ancillary is simply an unbiased estimator of zero plus a constant.). Then we have the following first-order version of Basu's theorem.
Theorem 1. A necessary and sufficient condition for a sufficient statistic $T$ to be boundedly complete is that every bounded first-order ancillary $V$ is uncorrelated (for all $\theta$ ) with every bounded real-valued function of $T$.

The proof is based on the following characterization of all bounded, unbiased estimators of zero.

Lemma 1 . If a sufficient statistic $T$ is boundedly complete, then a bounded statistic $S=S(X)$ is an unbiased estimator of zero if and only if (iff)

$$
\eta(t)=E[S \mid t]=0 \text { (a.e. } \mathscr{P}^{T} \text { ). }
$$

(Here (a.e. $\mathscr{P}^{T}$ ) means: except on a set $N$ of values of $T$, which has probability zero for all $\theta$.)

Proof of Lemma 1. If $S$ is an unbiased estimator of zero, then $E(S)=E[\eta(T)]=0$ for all $\theta$, and (3.1) follows from completeness. Conversely, if (3.1) holds, taking the expectation of both sides shows $S$ to be an unbiased estimator of zero.

Proof of Theorem 1. (a) Necessity. Suppose that $T$ is boundedly complete and that $V$ is a bounded first-order ancillary. Without loss of generality suppose that $E(V)$ $=0$. Then for any bounded $f(t)$ we have

$$
\operatorname{cov}_{\theta}(f(T), V)=E_{\theta}[f(T) V]=E_{\theta} \psi(T)
$$

where $\psi(t)=f(t) E[V \mid t]$. By the lemma, $E[V \mid t]$ and therefore $\psi(t)$ is zero (a.e. $\mathscr{P}^{T}$ ) and hence $\operatorname{cov}_{\theta}(f(T), V)$ $=0$ for all $\boldsymbol{\theta}$.

(b) Sufficiency. If $T$ is not boundedly complete, there exists a bounded function $f$ such that $E_{\theta} f(T)=0$ for all $\theta$ but $f(T) \neq 0$ with positive probability for some $\theta_{0}$. Put $V(X)=f(T)$. Then $\operatorname{cov}_{\theta_{0}}(V, f(T))=E_{\theta_{0}}\left[f^{2}(T)\right]>0$, and this proves the condition to be sufficient.

The analogous result holds for completeness instead of bounded completeness if attention is restricted to statistics with finite variance.

Instead of modifying the definition of ancillarity to obtain a converse of Basu's theorem, one can instead modify the definition of completeness. Quite generally, call a sufficient statistic $\mathscr{F}_{F}$-complete if $E_{\theta} f(T)=0$ for all $\theta$ and $f \in \mathscr{F}^{P}$ implies $f(t)=0$ (a.e. $\mathscr{P}^{T}$ ) where $\mathscr{F}$ is some given class of real-valued functions. Completeness and bounded completeness correspond respectively to the case that $F$ is the class of all integrable or all bounded functions. The case that it is the class of all square integrable functions is relevant for the analog of Theorem 1 mentioned following the proof of that theorem.

If $F_{0}$ is the class of all two-valued functions, we have the following result, which provides a partial converse of Basu's theorem.

\section{Theorem 2. If}

Every ancillary statistic is independent of $T$,

then $T$ is $F_{0}$-complete.

Proof. Suppose $T$ is not $F_{0}$-complete. Then there exists a two-valued function $f$ such that $E_{\theta} f(T)=0$ for all $\theta$, 
but $f$ is not zero with probability one. Let $f(t)=-a$ when $t \in A$ and $f(t)=b$ otherwise. Then $P_{\theta}(T \in A)$ $=b /(a+b)$ is independent of $\theta$ so that the indicator of $A, I_{A}(T)$, is an ancillary. This provides a contradiction.

Basu's theorem, together with Theorem 2, shows that Bounded completeness $\Rightarrow(3.2) \Rightarrow F_{0}$-completeness.

That neither of these implications holds in the other direction is shown by the following example.

\section{Example 2. Let $X$ be distributed as}

$$
\begin{array}{llllllllll}
-5 & -4 & -3 & -2 & -1 & 1 & 2 & 3 & 4 & 5
\end{array}
$$$$
\alpha^{\prime} p^{2} q \alpha^{\prime} p q^{2} \frac{1}{2} p^{3} \frac{1}{2} q^{3} \gamma^{\prime} p q \gamma p q \frac{1}{2} q^{3} \frac{1}{2} p^{3} \alpha p q^{2} \alpha p^{2} q
$$

where the first row lists the values $X$ takes on and the second row their probabilities and where $\alpha, \gamma, \alpha^{\prime}, \gamma^{\prime}$ are positive constants satisfying

$$
\alpha+\gamma=\alpha^{\prime}+\gamma^{\prime}=\frac{3}{2} .
$$

It is easy to check the following facts. to 1 .

I If $0<p<1, q=1-p$, the probabilities add up

II $T=|X|$ is minimal sufficient.

III $P(X>0)=\frac{1}{2}$ so that the indicator $V$ of the set $\{X>0\}$ is an ancillary statistic.

IV If $\alpha \neq \alpha^{\prime}, V$ is not independent of $T$, so that (3.2) does not hold.

$\mathrm{V}$ To see that $T$ is $F_{0}$-complete, note that $E f(T)=$ 0 iff

$$
\begin{aligned}
& f(1)\left[\gamma+\gamma^{\prime}\right] p q+f(2) q^{3}+f(3) p^{3} \\
& +\left(\alpha+\alpha^{\prime}\right)\left[f(4) p q^{2}+f(5) p^{2} q\right]=0 .
\end{aligned}
$$

This holds for all $0<p<1$ iff

$$
\begin{aligned}
& f(2)=f(3)=0, \quad f(1)=a, \\
& f(4)=f(5)=-\frac{\gamma+\gamma^{\prime}}{\alpha+\alpha^{\prime}} a
\end{aligned}
$$

for some $a$. Since no two-valued $f$ can satisfy (3.4), $T$ is $F_{0}$-complete.

It now follows from (IV) that $F_{0}$-completeness does not imply (3.2). Also, since (3.4) shows $T$ not to be boundedly complete, $F_{0}$-completeness does not imply bounded completeness.

That (3.2) does not imply bounded completeness can be seen from any example without bounded completeness in which no ancillary statistic exists and (3.2) therefore holds vacuously. A less trivial example is obtained by puting $\alpha=\alpha^{\prime}, \gamma=\gamma^{\prime}$ in Example 2. The totality of ancillary statistics is then obtained by selecting any of the 5 sign combinations $\pm 1, \pm 2, \pm 3, \pm 4, \pm 5$, for example $+1,-2,-3,+4,-5$ and setting

$V(+1)=V(-2)=V(-3)=V(4)=V(-5)=1$
For any such $V, P(V=1)=P(V=0)=\frac{1}{2}$ and $V$ is independent of $T$, so that (3.2) holds. On the other hand, $T$ has been seen not to be boundedly complete.

That $F_{0}$-completeness is a very weak (and somewhat unnatural) condition is indicated by the fact that a sufficient statistic can be $F_{0}$-complete with being minimal. As a simple example, suppose that $X$ and $Y$ are independent Poisson variables with $E(X)=E(Y)=\lambda$. Then $T=X+Y$ is minimal sufficient and complete, but $(X$, $Y$ ) is $F_{0}$-complete. This follows from the facts that the conditional distribution of $X$ given $X+Y=t$ is the binomial distribution $b\left(\frac{1}{2}, t\right)$ and that for no constant $c$ does there exist a nontrivial two-valued function $f(x, y)$ such that $E[f(X, Y) \mid t]=c$ for all $t$.

Example 2, together with (3.3), shows that bounded completeness is a stronger condition than (3.2) while $F_{0}$ completeness is not strong enough. It is interesting that there exists an intermediate completeness condition which is exactly equivalent to (3.2). Although the condition is rather intractable and not likely to be of much use, we give it here for the sake of completeness.

Let $F_{1}$ be the class of all functions $f(t)$ which are the conditional expectation of a two-valued function of $X$ given $t$. If $X=T$, then $F_{1}$ reduces to $F_{0}$.

Theorem 3. Condition (3.2) is necessary and sufficient for $T$ to be $F_{1}$-complete in the model $\mathscr{P}$.

Proof. (a) Suppose $T$ is $F_{1}$-complete with respect to $\mathscr{P}$ and that $V$ is an ancillary statistic. Then for any set $A$, the probability $P(V \in A)=p$ is independent of $\theta$, and $g(V)=I_{A}(V)-p$ is both a two-valued function of $X$ and an unbiased estimator of zero. If $f(t)=E[g(V) \mid t]$, it follows as in the proof of Theorem 1(a) that $E_{\theta} f(T)=0$ for all $\theta$. Since $f \in F_{1}$, this implies

$$
f(t)=P[V \in A \mid t]-p=0 \text { a.e. }
$$

and hence the independence of $V$ and $T$.

(b) Conversely, suppose that $T$ is not $F_{1}$-complete, so that there exists $f \in F_{1}$ which is not 0 a.e. but such that $E_{\theta} f(T)=0$ for all $\theta$. Let $g$ be a nontrivial two-valued function of $X$ for which $E[g(X) \mid t]=f(t)$, say $g(x)=a$ if $x \in A$ and $g(x)=b$ otherwise. Then $E_{\theta} g(X)=0$, the indicator $V$ of $A$ is an ancillary statistic as in the proof of Theorem 2 , but by the assumption about $f, V$ is not independent of $T$.

\section{INTERPRETATION OF COMPLETENESS}

Ancillary statistics by themselves have no value for estimating $\theta$ and the same is likely to be true of first-order ancillaries (since their expectation is independent of $\theta$ ), although statistics with either property may be valuable when used in conjunction with other statistics. The results of the preceding section can be summarized roughly by saying that the various forms of completeness of a sufficient statistics $T$ characterize the success of $T$ in separating the informative part of the data from that part which by itself carries no (or little) information. 
Basu's theorem explains how a complete sufficient statistic achieves this separation. It does this by making the ancillary part of the data independent of (or uncorrelated with) $T$. For the technical reasons mentioned in Section 2 , this interpretation of completeness is not as clear-cut as one might like. Roughly speaking, however, a sufficient statistic $T$ is complete if it contains no ancillary information or equivalently if all ancillary information is independent of (or at least uncorrelated with) $T$.

The idea that it is the lack of correlation between firstorder ancillaries and functions of $T$ that makes complete sufficient statistics so effective as a noise-free distillation of the data finds some support in a theorem of Lehmann and Scheffé (1950) and Rao (1952). Suppose $T$ is not complete, and consider the functions $f(T)$ which are uncorrelated with all first-order ancillaries (all functions being assumed to be square integrable). One might then expect these $f$ 's (though not sufficient) to be particularly effective estimators. The theorem in question states in part that these functions are the only unbiased estimators with uniformly minimum variance (UMVU).

Unfortunately, it turns out that in many common problems there are no functions $f$ for which the above property holds. For example, when $X_{1}, \ldots, X_{n}$ are iid according to the uniform distribution on $\left(\theta-\frac{1}{2}, \theta+\frac{1}{2}\right)$, it was shown by Lehmann and Scheffé $(1950,1956)$ that no function of $\theta$ has a UMVU estimator. One might expect this property to hold fairly generally in location problems with incomplete minimal sufficient statistic. (For some partial results in this direction see Bondessen 1975.) The corresponding result was proved by Unni (1978) for the case that $X_{1}, \ldots, X_{n}$ is a sample from the normal distribution $N\left(r \sigma, \sigma^{2}\right), r=$ known, and for the BehrensFisher problem (i.e., the case of samples from two normal distributions $N\left(\xi, \sigma^{2}\right), N\left(\xi, \tau^{2}\right)$ with unknown variances and common unknown mean.) Examples in which the minimal sufficient statistic is not complete but in which some parametric functions have UMVU estimators can of course be constructed (see for instance Ex. 5.2 of Lehmann and Scheffé 1950), but even then the dimension of the space of such functions is typically quite low.

\section{ANCILLARY STATISTICS AND THE PRINCIPLE OF CONDITIONALITY}

When a model is not complete, the minimal sufficient statistic will contain ancillary or at least first-order ancillary statistics, and as a result its dimension will typically be larger than that of the parameter space. The question then arises as to how to use the ancillary information to effect a further reduction of the data in order to facilitate the inference problem. Little is known about ways of utilizing first-order ancillaries (the theorems by Lehmann and Scheffé and by Rao mentioned in Section 4 can be viewed as a contribution in this direction) but there is considerable literature on the use of ancillary statistics. The ideas were first put forward by R.A. Fisher $(1934,1935)$ and an excellent discussion of the issues can be found in Cox and Hinkley (1974). Many additional results are given by Barndorff-Nielsen (1978). As a brief introduction to the subject, we shall here discuss a few examples.

Example 3. (a) In the standard introductory example, which is implicit in the writing of Fisher, the natural ancillary statistic is the sample size. To be specific, let $N$ be a random variable taking on values $1,2, \ldots$ with known probabilities $\pi_{1}, \pi_{2}, \ldots$. Having observed $N$ $=n$, we perform $n$ binomial trials with success probability $p$ and let $S_{n}$ be the number of successes in these trials. For the observation $X=\left(N, S_{N}\right), X$ is minimal sufficient and $N$ is ancillary.

The natural estimator $S_{N} / N$ of $p$ is unbiased and has variance $p q E(1 / N)$. It is, however, not clear that this is the appropriate measure of accuracy for the following reason. The most striking feature of the example is that $N$, while not providing any information about $p$, tells us how much information about $p$ is contained in $S_{N}$. If the observed value $n$ of $N$ is large, $S_{N}$ is highly informative; for small $n$ it carries little information. For this reason it may be better to cite as measure of accuracy the conditional variance $p q / n$ as appropriate in those cases in which $n$ has been obtained.

If this argument is accepted, not only the variance but the whole problem should be viewed conditionally. This includes in particular the choice of estimate which in the present case would remain unchanged but in others might not. From this point of view, conditioning on $N$ has the advantage of reducing the random part of the data from $\left(N, S_{N}\right)$ to the simple one-dimensional statistic $S_{n}$. Since $S_{n}$ is complete, the conditional model is complete, there exist UMVU estimators for $p$ and $p q / n$, and so on.

(b) Example (a) may seem artificial (although very similar situations arise in sampling from finite populations) but some of its principal features are in fact quite typical. Consider for instance once more the location problem of Example 1. Here the differences $Y_{i}$ provide the ancillary information which is analogous to $N$, and typically give an indication of the dispersion of the conditional distribution of an estimator of $\theta$ such as the mean or median. This is particuarly clear in the case of the uniform distribution. The minimal sufficient statistic can then be represented as $(Z, Y)$ with $Z=\frac{1}{2}\left[X_{(1)}+X_{(n)}\right]$ and $Y=X_{(n)}-X_{(1)}$. The conditional distribution of $Z$, given $Y=y$, is the uniform distribution on $\left(\theta-\frac{1}{2}(1-y), \theta\right.$ $\left.+\frac{1}{2}(1-y)\right)$. Thus $Y$ again measures the amount of information $Z$ contains about $\theta$ and it is natural to restrict attention to the conditional distribution of $Z$ given $Y$. In this case even the conditional model is not complete. (Additional ancillaries exist but are rather unsatisfactory. See Basu 1964, Sec. 4.)

(c) As a last illustration consider the ancillary statistic $V=I\{x>0\}$ of Ex. 2. The probabilities of the conditional distribution of $X$ given $V=1$ are

$$
\begin{array}{ccccc}
1 & 2 & 3 & 4 & 5 \\
(3-2 \alpha) p q, & q^{3}, & p^{3}, & 2 \alpha p q^{2}, & 2 \alpha p q^{2} .
\end{array}
$$


Given $V=0$, they are the corresponding values with $\alpha$ replaced by $\alpha^{\prime}$. It is easy to see that an experiment with outcome probabilities (5.1) is more informative than the corresponding experiment with $\alpha^{\prime}$ when $\alpha>\alpha^{\prime}$. (The reason is that the events with probabilities $2 \alpha p q^{2}$ and $2 \alpha p q^{2}$ can be split at random into four events with probabilities $2 \alpha^{\prime} p q^{2}, 2 \alpha^{\prime} p^{2} q, 2\left(\alpha-\alpha^{\prime}\right) p q^{2}, 2\left(\alpha-\alpha^{\prime}\right) p^{2} q$. The latter two have a combined probability of $2(\alpha-$ $\left.\alpha^{\prime}\right) p q$, which can be combined with $(3-2 \alpha) p q$ to yield an event with probability $\left(3-2 \alpha^{\prime}\right) p q$. Thus, from the $\alpha$-experiment and a table of random numbers one can generate the $\alpha^{\prime}$-experiment but not vice versa.)

The above considerations suggest the conditionality principle, first proposed by Fisher $(1935,1936,1956)$ and investigated as a fundamental principle by Birnbaum (1962): in the presence of an ancillary statistic $V$, statistical inference should be carried out not in the overall model but in the conditional model given $V$.

Apart from possible reservations which one may have about the principle itself, difficulties in implementing it arise from the fact that ancillary statistics are not unique. In Example 3(b), for instance, $\sum\left(X_{i}-\bar{X}\right)^{2}$ is ancillary, as is $\sum\left|X_{i}-\bar{X}\right|$ or any other function of the differences. To avoid this difficulty it is tempting, in analogy with the definition of a minimal sufficient statistic, to call an ancillary statistic $V$ maximal if every other ancillary statistic is a function of $V$. One would then condition on this maximal $V$, thereby carrying the conditioning process as far as possible.

Unfortunately, a maximal $V$ in this strong sense often does not exist. Basu (1964) has therefore introduced a weaker concept and has defined $V$ as maximal if there exists no ancillary statistic $V^{\prime}$ of which $V$ is a function. Such maximal ancillaries, however, frequently are not unique. In searching for a way out of the difficulty, it is helpful to recall the motivation for conditioning which was given in the comments after Example 3, and which was stated by Fisher (1935, p. 48) as follows: "Ancillary statistics are only useful when different samples of the same size can supply different amounts of information, and serve to distinguish those which supply more from those which supply less.",

Fisher's statement leads to the idea that the better an ancillary statistic is, in distinguishing the more informative samples from those that are less so, the more useful it is as a conditioning variable. An implementation of this criterion for choosing an ancillary has been provided by Cox (1971), who compares different ancillaries $V$ in terms of the variance of the Fisher information of the conditional distribution of $X$ given $V$. An ancillary $V$ is preferred to $V^{\prime}$ if it has the larger such variance for all parameter values. Such a principle will resolve the choice of ancillary in many cases although it may of course turn out that it leads to different choices for different parameter values.

If $X=(Y, V)$, where $V$ is ancillary, conditioning on $V$ can be thought of as data reduction obtained by replacing the random quantity $V$ by a known constant $v$, so that part of the randomness of the data is eliminated. It frequently happens that the conditional model for $Y$ given $v$ is complete, in which case conditioning is particularly rewarding. It is interesting to note that in this latter case the ancillary $V$ is essentially maximal in the sense of Basu (1959). This result (to which we hope to return in a later paper) presents a considerable strengthening of Theorem 7 of Basu (1959).

In summary it is seen that models involving ancillaries contained in the minimal sufficient statistic are more difficult to analyze than complete models. Even in so relatively simple a situation as that of Example 1, no general agreement has been reached whether to assess the accuracy of the best estimator of $\theta$ (the Pitman estimator) conditionally or unconditionally. The situation becomes even more complicated when nuisance parameters are present, a possibility we have not considered here.

Commenting on his efforts to develop a satisfactory small-sample theory of estimation, Fisher wrote in 1956 (pp. 157 and 158): "The most important step which has been taken so far to complete the structure of the theory of estimation is the recognition of Ancillary statistics." Despite some important recent work on conditional inference by Efron and Hinkley (1978) and Hinkley (1977), we still seem to be far from a full understanding of this difficult topic and a satisfactory small-sample theory of models that lack completeness.

\section{[Received November 1979. Revised December 1980.]}

\section{REFERENCES}

BAHADUR, R.R. (1954), "Sufficiency and Statistical Decision Functions," Annals of Mathematical Statistics, 25, 423-462.

BARNARD, G.A., and SPROTT, D.A. (1971), "A Note on Basu's Examples of Anomalous Ancillary Statistics" (with discussion), in Foundations of Statistical Inference, eds. Godambe and Sprott, 163-176. Toronto: Holt, Rinehart and Winston.

BARNDORFF-NIELSEN, O. (1978), Information and Exponential Families in Statistical Theory, New York: John Wiley.

BASU, D. (1955, 1958), "On Statistics Independent of a Complete Sufficient Statistic," Sankhyā, 15, 377-380 and 20, 223-226.

(1959), "The Family of Ancillary Statistics,"' Sankhyä, 21, 247-256

(1964), "Recovery of Ancillary Information," Sankhyā, Ser. A, 26, 3-16.

BIRNBAUM, A. (1962), "On the Foundations of Statistical Inference" (with discussion), Journal of the American Statistical Association, 57, 269-306.

BONDESSEN, L. (1975), “Uniformly Minimum Variance Estimation in Location Parameter Families," Annals of Statistics, 3, 637-660. COX, D.R. (1971), "The Choice Between Alternative Ancillary Statistics," Journal of the Royal Statistical Society, Ser. B, 33, 251-255. COX, D.R., and HINKLEY, D.V. (1974), Theoretical Statistics, London: Chapman and Hall.

EFRON, B., and HINKLEY, D.V. (1978), "Assessing the Accuracy of the Maximum Likelihood Estimator: Observed Versus Expected Fisher Information"' (with comments), Biometrika, 65, 457-487.

FISHER, R.A. (1934), "Two New Properties of Mathematical Likelihood," Proceedings of the Royal Statistical Society, Ser. A, 144, 285-307.

(1935), "The Logic of Inductive Inference." Journal of the Royal Statistical Society, 98, 39-54.

(1936), "Uncertain Inference," Proceedings of the American Academy of Arts and Science, 71, No. 4, 245-258.

(1956), Statistical Methods and Scientific Inference, New York:

Hafner. 
HINKLEY, D.V. (1977), "Conditional Inference About a Normal Mean With Known Coefficient of Variation," Biometrika, 64, 105-108.

LANDERS, D., and ROGGE, L. (1972), "Minimal Sufficient $\sigma$-Fields and Minimal Sufficient Statistics. Two Counterexamples." Annals of Mathematical Statistics, 43, 2045-2049.

LEHMANN, E.L., and SCHEFFÉ, H. (1950, 195, 1956), "Completeness, Similar Regions, and Unbiased Estimation,"'Sankhyā, 10 , 305-340; 15, 219-236; and 17, 250.
PITCHER, T.S. (1975), "Sets of Measures Not Admitting Necessary and Sufficient Statistics or Subfields," Annals of Mathematical Statistics, 26, 267-268

RAO, C.R. (1952), "On Statistics With Uniformily Minimum Variance," Science and Culture, 17, 483-484.

UNNI, K. (1978), "The Theory of Estimation in Algebraic and Analytic Exponential Families With Applications to Variance Component Models,'” Ph.D. thesis, Indian Statistical Institute, Calcutta. 